\title{
Calidad de los hábitos alimentarios (adherencia a la dieta mediterránea) en los alumnos de educación secundaria obligatoria
}

\author{
Quality of dietary habits (adherence to a mediterranean diet) in \\ pupils of compulsory secondary education
}

\section{A. Ayechu, T. Durá}

\section{RESUMEN}

Fundamento. La dieta mediterránea está considerada como un prototipo de dieta saludable. La modernización de la sociedad implica cambios culturales y/o sociológicos que afectan a los hábitos y preferencias alimentarias. El objetivo de este trabajo es determinar el índice de calidad de los hábitos alimentarios entre los alumnos de educación secundaria obligatoria (ESO).

Material y métodos. Distribución de un cuestionario de 16 preguntas (test kidmed) a una muestra aleatoria de 1.956 alumnos de ESO (966 varones y 990 mujeres). La puntuación final o índice kidmed (de 0 a 12) indica si la adherencia a la dieta mediterránea es baja (de 0 a 3), media (de 4 a 7 ) o alta (de 8 a 12).

Resultados. El 6,7\% de los alumnos de ESO tenían un índice kidmed bajo, el 50,4\% medio y el $42,9 \%$ alto, sin que existieran diferencias significativas entre ambos sexos. El índice kidmed disminuía progresivamente con la edad ( $\mathrm{p}<0,05)$. A los 13 años ( $\left.1^{\circ} \mathrm{ESO}\right)$ el 49,5\% tenían una puntuación óptima del índice kidmed, mientras que a los 16 años ( $\left.4^{\circ} \mathrm{ESO}\right)$ esta puntuación apenas la alcanzaba un $37,2 \%$. Entre los alumnos de $1^{\circ}$ y $4^{\circ}$ de ESO existían diferencias significativas $(\mathrm{p}<0,05)$ respecto al consumo de frutas, pescados, frutos secos, bollería industrial y dulces; así como en la falta de regularidad del desayuno y la frecuentación de hamburgueserías.

Conclusiones. Al terminar la ESO el 62,8\% de los alumnos presentaban una adherencia media-baja a la dieta mediterránea. Las normas dietéticas aplicables a estos adolescentes consistirían en incrementar el consumo de frutas, verduras y hortalizas, frutos secos, pastas y arroz, yogures y quesos, legumbres y pescados, así como disminuir la ingesta de bollería industrial y dulces y la frecuentación de hamburgueserías; siendo importante el desayuno diario y el consumo de aceite de oliva como grasa culinaria. Sería conveniente desarrollar programas de educación nutricional durante la enseñanza obligatoria.

Palabras clave. Adolescentes. Dieta mediterránea. Hábitos dietéticos.

An. Sist. Sanit. Navar. 2010; 33 (1): 35-42

\begin{abstract}
Background. The Mediterranean diet is considered to be a prototype of a healthy diet. The modernization of society implies sociological and cultural changes that affect feeding preferences and habits. The aim of this paper is to determine the quality index of the dietary habits in pupils of compulsory secondary education (CSE).
\end{abstract}

Material and methods. By distributing a 16 item questionnaire (kidmed test) to a random sample of $1,956 \mathrm{pu}-$ pils of CSE ( 966 males and 990 females). The final score or kidmed index (range 0-12) indicates whether the degree of adherence to the Mediterranean diet is low (0-3), medium (4-7) or high (8-12).

Results. A low kidmed index value was registered in $6.7 \%$ of the pupils of CSE, whereas medium values corresponded to $50.4 \%$ and low values to $42.9 \%$, showing no significant statistical differences between the sexes. The kidmed index decreases progressively with age $(\mathrm{p}<0.05)$. At age 13 (first year of CSE), $49.5 \%$ of the pupils had an optimal score on the kidmed index, whereas at 16 (fourth year of CSE), this score reached $37.2 \%$. There were statistical differences $(\mathrm{p}<0.05)$ regarding consumption of fruit, fish, nuts and dried foods, sweets and factory produced baked foodstuffs; pupils also frequently went without breakfast and ate at fast food restaurants.

Conclusions. By the end of CSE, $62.8 \%$ of pupils showed a low-medium degree of adherence to the Mediterranean diet. Applicable food advice for these adolescents would be to increase consumption of fruit, vegetables, nuts, pasta and rice, yogurt and cheese, pulses and fish; and to reduce consumption of factory produced baked foodstuffs and sweets and to eat less at fast food restaurants; besides insisting on the importance of daily breakfast and the use olive oil for cooking. In addition, it would be convenient to develop nutrition education programs during compulsory education.

Key words. Adolescents. Mediterranean diet. Dietary habits.

\section{Correspondencia}

Teodoro Durá Travé

Avda. Pío XII, $10-8^{\circ} \mathrm{C}$

31008 Pamplona

E-mail:tduratra@cfnavarra.es 


\section{INTRODUCCIÓN}

El patrón dietético tradicionalmente observado en los países mediterráneos ha dado lugar al concepto de dieta mediterránea, caracterizada por un alto consumo de verduras y hortalizas, legumbres, frutas, frutos secos y cereales y, especialmente, aceite de oliva; junto con un consumo moderado de pescados, huevos y productos lácteos, preferentemente yogur o queso, y un menor consumo de carnes y grasas animales ${ }^{1-4}$. La dieta mediterránea está considerada como un prototipo de dieta saludable, ya que garantiza un aporte calórico y de nutrientes en cantidades suficientes y proporciones adecuadas $^{5-8} ; \mathrm{y}$, además, contribuye a la prevención de enfermedades cardiovasculares, hipertensión, diabetes, cáncer, etc. y, en general, a una mayor esperanza de vida ${ }^{4,9-14}$.

La modernización de la sociedad implica una serie de cambios culturales y/o sociológicos que afectan inevitablemente a los hábitos y preferencias alimentarias. Cada vez se dedica menos tiempo a la compra de alimentos y elaboración de comidas, con una alternativa evidente: alimentos procesados fáciles de preparar y rápidos de consumir que, generalmente, conllevan un consumo excesivo de alimentos de origen animal, especialmente de carnes y derivados, y de azúcares refinados, con el consecuente incremento de grasas saturadas y colesterol en la dieta ${ }^{8,15-19}$.

Este virtual deterioro de los patrones alimentarios tradicionales en nuestra población tiene lugar especialmente en aquellos sectores más susceptibles de ser influidos por el entorno como, por ejemplo, los niños y adolescentes ${ }^{16,20-23}$, que, por otro lado, serían los más necesitados de una alimentación saludable para un crecimiento y desarrollo óptimos. Por tanto, la población adolescente constituiría potencialmente un grupo de riesgo nutricional que justificaría el estudio de la calidad de sus hábitos alimentarios

El objetivo del presente trabajo consiste en determinar el índice de calidad de los hábitos alimentarios (adherencia a la dieta mediterránea) entre los alumnos de educa- ción secundaria obligatoria de la ciudad de Pamplona.

\section{MATERIAL Y MÉTODOS}

El municipio de Pamplona comprende una población total de 183.964 habitantes (censo 2001, Instituto de Estadística de Navarra) de los que 6.179 (3,36\%) formaban en el año 2007 el grupo de población con edades comprendidas entre 13 y 16 años y/o de cursar educación secundaria obligatoria (ESO). El marco muestral considerado lo formaron estos 6.179 adolescentes (3.145 varones y 3.034 mujeres). Para calcular el tamaño de la muestra se ha tomado la hipótesis más desfavorable $(0,50)$, un nivel de confianza del $99 \%(\alpha=0,01)$ y una precisión del 0,03 , resultando el número mínimo necesario de casos de 1.419 alumnos $^{24}$.

Para valorar la calidad de los hábitos alimentarios (adherencia a un patrón dietético mediterráneo) se utilizó el test de adhesión a la dieta mediterránea kidmed aplicado con anterioridad, y de manera satisfactoria, en el mismo grupo de población $^{25,26}$. La muestra se obtuvo mediante la distribución de un cuestionario o test kidmed, en un día lectivo, a la totalidad de alumnos de ESO de seis centros públicos y/o concertados, seleccionados de manera aleatoria, de la ciudad de Pamplona, en el segundo trimestre del curso académico 2007-2008. Todos los alumnos que habían acudido a sus distintos centros académicos el día previsto para la distribución del cuestionario $(\mathrm{n}=1.980)$ aceptaron su participación; no obstante fueron rechazados 24 cuestionarios por cumplimentación errónea y/o insuficiente. Por tanto, la tasa de participación podría considerarse del $98,8 \%$.

El total de participantes fueron 1.956 alumnos: 966 varones $(49,4 \%)$ y 990 mujeres $(50,6 \%)$, cuya distribución por cursos y sexo fue la siguiente: $1^{\circ}$ ESO $(n=517), 256$ varones y 261 mujeres; $2^{\circ}$ ESO $(n=530), 260$ varones y 270 mujeres; $3^{\circ}$ ESO $(n=447), 240$ varones y 207 mujeres, y $4^{\circ}$ ESO $(n=462)$, 210 varones y 252 mujeres. Para la comparación de proporciones entre los distintos 
cursos de ESO se ha establecido un riesgo del 0,01 (potencia del 99\%).

El test kidmed (Tabla 1) consiste en un cuestionario de 16 preguntas que deben responderse de manera afirmativa/negativa (sí/no). Las respuestas afirmativas en las preguntas que representan una connotación negativa en relación con la dieta mediterránea (son 4) valen -1 punto, y las respuestas afirmativas en las preguntas que representan un aspecto positivo en relación con la dieta mediterránea (son 12) valen +1 punto. Las respuestas negativas no puntúan. Por tanto, dicho índice puede oscilar entre 0 (mínima adherencia) y 12 (máxima adherencia).

Tabla 1. Test kidmed ${ }^{25}$.

\begin{tabular}{lc}
\multicolumn{1}{c}{ Test de calidad de la dieta mediterránea } & \\
\hline Toma una fruta o zumo de fruta todos los días & +1 \\
Toma una segunda fruta todos los días & +1 \\
Toma verduras frescas o cocinadas una vez al día & +1 \\
Toma verduras frescas o cocinadas más de una vez al día & +1 \\
Toma pescado por lo menos 2 ó 3 veces a la semana & +1 \\
Acude una vez o más a la semana a una hamburguesería & -1 \\
Toma legumbres más de 1 vez a la semana & +1 \\
Toma pasta o arroz casi a diario (5 o más veces por semana) & +1 \\
Desayuna un cereal o derivado (pan, tostadas,...) & +1 \\
Toma frutos secos por lo menos 2 ó 3 veces a la semana & +1 \\
En su casa utilizan aceite de oliva para cocinar & +1 \\
No desayuna todos los días & -1 \\
Desayuna un lácteo (leche, yogur, etc.) & +1 \\
Desayuna bollería industrial & -1 \\
Toma 2 yogures y/o queso (40 g) todos los días & +1 \\
Toma dulces o golosinas varias veces al día & -1 \\
\hline
\end{tabular}

La suma de los valores de dicho cuestionario da lugar al índice kidmed, que se clasifica en tres categorías:

1. De 8 a 12: dieta mediterránea óptima (adherencia alta)

2. De 4 a 7 : necesidad de mejora en el patrón alimentario para adecuarlo al modelo mediterráneo (adherencia media).

3. De 0 a 3: dieta de muy baja calidad (adherencia baja).

Los resultados se expresan como porcentajes. El análisis estadístico (Chi-cua- drado) fue realizado mediante el programa informático SPSS 17.0 para windows.

\section{RESULTADOS}

En la tabla 2 se exponen y comparan los resultados del test kidmed entre ambos sexos. El $6,7 \%$ de la totalidad de la muestra tenían un valor del índice kidmed muy bajo, un $50,4 \%$ intermedio y un $42,9 \%$ alto, sin que existieran diferencias significativas entre ambos sexos. Sin embargo, existían diferencias estadísticamente significativas $(\mathrm{p}<0,05)$ entre distintos ítems: 
los varones acudían una vez o más a la semana a una hamburguesería, tomaban pasta o arroz casi a diario (5 o más veces por semana), desayunaban cereales o derivados (pan, tostadas, etc.), tomaban frutos secos por lo menos 2 ó 3 veces a la semana y desayunaban bollería industrial con mayor frecuencia que las mujeres; mientras que las mujeres tomaban verduras frescas o cocinadas una vez al día y no desayunaban todos los días con mayor frecuencia que los varones.

Tabla 2. Test de calidad de la dieta mediterránea en los alumnos de ESO por sexos.

\begin{tabular}{|c|c|c|c|}
\hline Test kidmed & Varones (\%) & Mujeres (\%) & Total (\%) \\
\hline Toma una fruta o zumo todos los días & 81,6 & 83,5 & 82,6 \\
\hline Toma una segunda fruta todos los días & 38,9 & 40,6 & 39,8 \\
\hline Toma verduras una vez al día $\left(^{*}\right)$ & 74,5 & 79,9 & 77,2 \\
\hline Toma verdura más de una vez al día & 26,2 & 27,3 & 26,7 \\
\hline Toma pescado con regularidad & 66,4 & 66,7 & 66,5 \\
\hline Acude con frecuencia a un fast-food $\left({ }^{*}\right)$ & 12,0 & 7,7 & 9,8 \\
\hline Toma legumbres con regularidad & 74,4 & 72,8 & 73,6 \\
\hline Toma pasta o arroz casi a diario $\left(^{*}\right)$ & 46,8 & 36,3 & 41,5 \\
\hline Desayuna un cereal o derivado $\left({ }^{*}\right)$ & 79,6 & 73,3 & 76,4 \\
\hline Toma frutos secos con regularidad $\left({ }^{*}\right)$ & 45,7 & 34,3 & 39,9 \\
\hline En casa utilizan aceite de oliva & 72,5 & 73,8 & 73,2 \\
\hline No desayuna todos los días $\left(^{*}\right)$ & 13,0 & 21,5 & 17,3 \\
\hline Desayuna un lácteo (leche, yogur, etc.) & 90,9 & 88,8 & 89,8 \\
\hline Desayuna bollería industrial $\left(^{*}\right)$ & 23,1 & 15,1 & 19,0 \\
\hline Toma 2 yogures y/o queso cada día & 53,1 & 51,7 & 52,4 \\
\hline Toma dulces varias veces al día & 18,3 & 16,5 & 17,4 \\
\hline \multicolumn{4}{|l|}{ Índice kidmed } \\
\hline$<3$ & 6,3 & 7,2 & 6,7 \\
\hline $4-7$ & 49,5 & 51,1 & 50,4 \\
\hline$>8$ & 44,2 & 41,7 & 42,9 \\
\hline
\end{tabular}

(*) $\mathrm{p}<0,05$ (Chi-cuadrado)

En la tabla 3 se exponen y comparan los resultados del test kidmed por cursos académicos y/o edad, objetivándose una disminución progresiva del índice kidmed con la edad. A los 13 años (1º ESO) el 49,5\% tenían un valoración óptima del índice kidmed, mientras que a los 16 años (4ํㅡㄹ esta valoración óptima la tenían un $37,2 \%$. Entre los alumnos de $1^{\circ}$ de ESO y los de $4^{\circ}$ de ESO se objetivaban diferencias significativas $(p<0,05)$ en el consumo de una segunda fruta diaria $(44,1 \%$ frente a $32 \%)$, de pescado por lo menos 2 ó 3 veces a la semana $(73,5 \%$ frente a $58,7 \%)$, de frutos secos por lo menos 2 ó 3 veces a la semana (43,3\% frente a $38,5 \%)$, de bollería industrial en el desayuno $(15,1 \%$ frente a $22,5 \%)$ y de dulces varias veces al día (13\% frente a $19,5 \%)$; así como en la falta de regularidad del desayuno (12,2\% frente a $17,1 \%)$ y la frecuentación de hamburgueserías $(6,4 \%$ frente a $10,4 \%$ ). 
Tabla 3. Test de calidad de la dieta mediterránea en los alumnos de ESO por curso académico.

\begin{tabular}{|c|c|c|c|c|}
\hline Test kidmed & $1^{\circ}$ ESO (\%) & $2^{\circ}$ ESO (\%) & $3^{\circ}$ ESO (\%) & $4^{\circ}$ ESO (\%) \\
\hline Toma una fruta o zumo todos los días & 84,1 & 81,7 & 83,7 & 80,7 \\
\hline Toma una segunda fruta todos los días $\left(^{*}\right)$ & 44,1 & 42,5 & 39,6 & 32,0 \\
\hline Toma verduras una vez al día & 80,7 & 75,5 & 76,5 & 76,2 \\
\hline Toma verdura más de una vez al día & 27,5 & 29,2 & 22,8 & 26,8 \\
\hline Toma pescado con regularidad $\left(^{*}\right)$ & 73,5 & 67,5 & 65,3 & 58,7 \\
\hline Acude con frecuencia a un fast-food $\left(^{*}\right)$ & 6,4 & 12,1 & 10,5 & 10,4 \\
\hline Toma legumbres con regularidad & 74,7 & 68,5 & 75,4 & 76,6 \\
\hline Toma pasta o arroz casi a diario & 41,5 & 44,5 & 43,4 & 39,6 \\
\hline Desayuna un cereal o derivado & 78,1 & 78,3 & 73,6 & 75,1 \\
\hline Toma frutos secos con regularidad $\left(^{*}\right)$ & 43,3 & 41,9 & 35,1 & 38,5 \\
\hline En casa utilizan aceite de oliva & 73,8 & 72,8 & 72,6 & 73,5 \\
\hline No desayuna todos los días $\left(^{*}\right)$ & 12,2 & 20,6 & 19,7 & 17,1 \\
\hline Desayuna un lácteo (leche, yogur, etc.) & 90,5 & 88,9 & 89,3 & 90,7 \\
\hline Desayuna bollería industrial $\left({ }^{*}\right)$ & 15,1 & 19,8 & 19,0 & 22,5 \\
\hline Toma 2 yogures y/o queso cada día & 55,1 & 50,2 & 50,1 & 54,1 \\
\hline Toma dulces varias veces al día (*) & 13,0 & 19,8 & 17,4 & 19,5 \\
\hline \multicolumn{5}{|l|}{ Índice kidmed $\left({ }^{*}\right)$} \\
\hline$<3$ & 4,8 & 8,3 & 7,4 & 6,5 \\
\hline $4-7$ & 45,7 & 46,4 & 54,1 & 56,3 \\
\hline$>8$ & 49,5 & 45,3 & 38,5 & 37,2 \\
\hline
\end{tabular}

(*) $\mathrm{p}<0,05$ (Chi-cuadrado)

\section{DISCUSIÓN}

Para evaluar el grado de adherencia a la dieta mediterránea se han elaborado distintas escalas de valoración, basadas en aspectos cualitativos y/o cuantitativos del consumo de los diferentes componentes «típicos» de la dieta mediterránea ${ }^{7,18,27-30}$. Estos índices de calidad de la dieta mediterránea se han utilizado exclusivamente en la población adulta y requieren un laborioso y complejo proceso de la información recogida sobre el consumo de alimentos. Actualmente se dispone del test kidmed, muy fácil de cumplimentar por parte del encuestado e interpretar por parte del encuestador, elaborado a partir de los índices citados y/o principios que sustentan el patrón alimentario mediterráneo, pero adaptado a la edad pediátrica y que permite evaluar de manera rápida la calidad de los hábitos alimentarios y, en concreto, determinar el grado de adherencia a la dieta mediterránea ${ }^{25}$. El índice kidmed constituye un instrumento que, por una parte, permite identificar de manera inmediata la población con hábitos alimentarios poco saludables; y, por otra parte, se ha constatado que una mayor puntuación garantiza un aporte de nutrientes en cantidades suficientes y proporciones adecuadas, lo que justificaría su empleo ${ }^{31}$. 
$\mathrm{Al}$ analizar las respuestas de los distintos ítems se advierte que tan sólo el 42,9\% de los alumnos encuestados reflejaban unos hábitos alimentarios compatibles con el patrón dietético mediterráneo (alta adherencia). Es decir, la mayoría de los alumnos de ESO (el 57,1\% restante) presentaban una adherencia media y/o baja y, por tanto, la necesidad de mejorar su patrón alimentario para adecuarlo al prototipo mediterráneo. En líneas generales, cabe destacar que los adolescentes encuestados no alcanzaban las recomendaciones de consumo de la mayoría de los alimentos que constituyen la base de la pirámide de la alimentación mediterránea; es decir, de frutas (apenas el 39,8\% tomaba diariamente una segunda pieza), de verduras y hortalizas (apenas el $26,7 \%$ tomaban diariamente una segunda ración), de frutos secos (apenas el 39,9\% los tomaban durante la semana) y de pasta o arroz (apenas el 41,5\% los tomaban casi a diario); y, además, el consumo de yogures y/o queso también era proporcionalmente bajo. Por otro lado, también cabe subrayar como un porcentaje relativamente importante de adolescentes desayunaban bollería industrial (el 19\% de los encuestados) o tomaban dulces diariamente (el 17,4\% de los encuestados); y conviene advertir que estos alimentos ocupan la cúspide de la pirámide nutricional y, por tanto, su frecuencia de consumo debería ser esporádica. La dieta mediterránea, al mismo tiempo que un prototipo de dieta saludable, representa un estilo de vida circunscrito a un marco climático y/o geográfico determinado ${ }^{2-5,18}$; $\mathrm{y}$, en este sentido, la tendencia de los adolescentes a frecuentar los establecimientos de comida rápida (el 9,8\% de los encuestados lo hacía una o más veces a la semana) y/o la falta de regularidad en el desayuno (el 17,3\% de los encuestados no desayunan diariamente) denotan, en gran medida, una perdida del legado cultural que representa la dieta mediterránea. El aceite de oliva constituye un elemento esencial de la cultura mediterránea que indefectiblemente prevalece en las diferentes etnias y/o países mediterráneos actuales y que, en gran medida, es responsable de los efectos beneficiosos atribuidos a este patrón dieté- tico ${ }^{4,32}$; lo que explicaría, en gran medida, el consumo culinario del aceite de oliva en sus domicilios manifestado mayoritariamente por los encuestados ${ }^{18,29}$.

Cuando se analiza el índice kidmed obtenido en relación con la edad y/o curso de ESO se observa un deterioro progresivo de la adherencia a la dieta mediterránea: mientras el 49,5\% de los alumnos del primer curso tenían una valoración óptima del índice kidmed (alta adherencia), al llegar al cuarto curso apenas un $37,2 \%$ alcanzaban dicha valoración. Este deterioro -especialmente derivado de un menor consumo de frutas y verduras, pescados y frutos secos y un mayor consumo de bollería industrial y dulces, así como de una menor regularidad en el desayuno y una mayor frecuentación de establecimientos de comida rápidapone de manifiesto una tendencia entre los alumnos de ESO a incorporarse a los nuevos patrones occidentales de hábitos alimentarios, con una producción y consumo cada vez mayor de alimentos procesados en detrimento de la cocina mediterránea basada en una amplia diversidad de alimentos naturales y frescos. Al terminar la educación obligatoria el $62,8 \%$ de los alumnos, sin diferencias entre sexos, presentaban una adherencia media y/o baja a la dieta mediterránea como consecuencia de la pérdida progresiva de unos hábitos dietéticos tradicionales de nuestro entorno geográfico que, por un lado, permitiría considerar que en una proporción importante de adolescentes existiría cierto riesgo de padecer alguna carencia y/o desequilibrio nutricional ${ }^{26} \mathrm{y}$, por otra parte, hace temer sobre una virtual desaparición de la dieta mediterránea a corto y/o medio plazo ${ }^{23,33,34}$.

De los resultados obtenidos se desprende la necesidad que tiene la población en general, y más en concreto los adolescentes, de una educación nutricional. Los adolescentes deben aprender que la dieta mediterránea, como prototipo de alimentación saludable, contribuye al mantenimiento de un óptimo estado de salud y que, aunque incluye todos los alimentos, su frecuencia de consumo debe seguir las pautas indicadas en la pirámide nutricio- 
nal. Por tanto, las normas dietéticas aplicables a estos adolescentes consistirían básicamente en incrementar el consumo diario de fruta fresca, verduras y hortalizas frescas y/o crudas, pastas y/o arroz, frutos secos (avellanas, almendras, nueces, cacahuetes, piñones, etc.), leche y derivados, principalmente yogur y/o queso, así como legumbres y pescados por lo menos 2 ó 3 veces por semana, además de fomentar el consumo de aceite de oliva como única grasa culinaria. Por otro lado, habría que recomendar el consumo ocasional de bollería industrial y dulces; además de insistir en la importancia de un desayuno diario que incluya cereales, lácteos y frutas ${ }^{35,36}$. No obstante, conviene advertir que este estudio adolece de una serie de limitaciones metodológicas, ya que no se registraron variables antropométricas y/o estilos de vida de los encuestados (status socioeconómico y/o nivel de estudio familiar, práctica de actividad física, hábitos televisivos, etc.) que podrían condicionar, en cierta medida, el grado de adherencia a la dieta mediterránea ${ }^{18,29}$.

En suma, sería conveniente diseñar programas de educación nutricional que, teniendo en cuenta el contexto social y/o familiar, tuvieran como objetivo conseguir que los adolescentes estuvieran en condiciones de llevar a cabo una alimentación saludable. Para ello, los poderes públicos tendrían que coordinar suficientes recursos humanos y/o materiales para intentar mantener nuestros hábitos dietéticos tradicionales y hacerlos compatibles con las nuevos estilos de vida de las sociedades modernas, promoviendo el consejo dietético en los programas de atención primaria y desarrollando programas de alimentación y nutrición en la enseñanza reglada; de tal modo que cuando los adolescentes terminaran su enseñanza obligatoria dispusieran de un excelente instrumento para prevenir la enfermedad y promover la salud.

\section{BIBLIOGRAFÍA}

1. Helsing E. Traditional diets and disease patterns of the mediterranean, circa 1960. Am J Clin Nutr 1995; 61 (Suppl): 1329-1337.
2. Willet WC, Sacks F, Trichopoulou A, Drescher G, Ferro-Luzzi A, Helsing E. Mediterranean diet pyramid: a cultural model for healthy eating. Am J Clin Nutr 1995; 61 (Suppl): 1402-1406.

3. TRichopoulou A, LAgiou P. Healthy traditional mediterranean diet: an expression of culture, history, and lifestyle. Nutr Rev 1997; 55: 383389.

4. Díaz I, Gascón E, Lázaro S, Maximiano C. Guía de la alimentación mediterránea. Ed. Empresa pública desarrollo agrario y pesquero. Consejería de agricultura y pesca. Junta de Andalucía 2007.

5. Sánchez-Villegas A, Bes-Rastrollo M, MartínezGonzÁlez, JA, Serra-Majem L. Adherence to a mediterranean dietary pattern and weight gain in a follow-up study: the SUN cohort. Int J Obes 2006; 30: 350-358.

6. Hu FB. The Mediterranean diet and mortality - olive oil and beyond. New Engl J Med 2003; 348: 2595-2596.

7. Trichopoulou A, Costacou T, Christina B, Trichopoulou D. Adherence to a mediterranean diet and survival in a greek population. New Engl J Med 2003; 348: 2599-2608.

8. Tur JA, Serra-Majem L, Romaguera D, Pous A. Does the diet of the Balearic population, a mediterranean type diet, still provide adequate antioxidant nutrient intakes? Eur J Nutr 2005, 44: 204-213.

9. Kris-Etherton P, Eckel RH, Howard BV, ST JeOr S, BAzzARRE TL. Lyon diet heart study. Benefits of a mediterranean-style, national cholesterol education program/american heart association step I dietary pattern on cardiovascular disease. Circulation 2001; 103: 1823-1825.

10. Ferrara LA, Raimondi S, d`Episcopo L, Guilda L, Dello Russo A, Marotta T. Olive oil and reduced need for antihypertensive medications. Arch Intern Med 2000; 160: 837-842.

11. Estruch R, Martínez-González MA, Corella D, Salas-Salvadó J, Ruiz-Gutiérrez V, Covas MI et al. PREDIMED study investigators. Effects of a mediterranean-style diet on cardiovascular risk factors: a randomized trial. Ann Intern Med 2006; 145: 1-11.

12. Hu FB, Rimm EB, Stampfer MJ, Ascherio A, Spiegelman D, Willet WC. Prospective study of major dietary patterns and risk of coronary heart disease in men. Am J Clin Nutr 2000; 72: 912-921.

13. Terry P, Hu FB, Hansen H, Wolk A. Prospective study of major dietary patterns and colorectal risk in women. Am J Epidemiol 2001; 154: 1143-1149. 
14. Trichopoulou A, Naska A, Antoniou A, Friel S, Trygg K, Turrini A. Vegetable and fruit: the evidence in their favour and the public health perspective. Int J Vitam Nutr Res 2003; 73: 63-69.

15. Rodríguez F, Banegas JR, Graciani MA, Hernández R, REY J. El consumo de alimentos y nutrientes en España en el período 1940-1988. Análisis de su consistencia con la dieta mediterránea. Med Clin (Barc) 1996; 106: 161-168.

16. DURÁ T. Ingesta de energía y nutrientes en los alumnos de educación secundaria obligatoria. An Esp Pediatr 2001; 54: 547-554.

17. Aranceta J. Spanish food patterns. Public Health Nutr 2001; 4: 1399-1402.

18. Sánchez-Villegas A, Martínez JA, De Irala J, MarTíNEZ-GonzÁLEz MA. Determinants of the adherence to an "a priori» defined mediterranean dietary pattern. Eur J Nutr 2002; 41: 249-257.

19. Moreno LA, SArría A, Popkin BM. The nutrition transition in Spain: a european mediterranean country. Eur J Clin Nutr 2002; 56: 9921003.

20. Iturbe A, Emparanza J, Perales A. Modelo dietético de los adolescentes de Guipúzcoa. An Esp Pediatr 1999; 50: 471-478.

21. Samuelson G, Bratteby LE, Enghardt H, Hedgren M. Food habits and energy and nutrient in swedish adolescents approaching the year 2000. Acta Paediatr 1996 (Suppl 415): 1-20.

22. Serra-Majem L, García-Closas R, Ribas L, PérezRodrigo C, Aranceta J. Food patterns of spanish schoolchildren and adolescents: The enKid study. Public Health Nutrition 2001; 4 : 1433-1438.

23. Tur JA, Romaguera D, Pons A Food consumption patterns in a mediterranean region: does the mediterranean diet still exist? Ann Nutr Metab 2004; 48: 193-201.

24. Fernández P. Determinación del tamaño muestral. Cad Aten Primaria 1996; 3: 138-141 (actualización 06/03/2001).

25. Disponible en: http://www.fisterra.com

26. Serra-Majem L, Ribas L, Ngo J, Ortega RM, García A, Pérez-Rodrigo $\mathrm{C}$ et al. Food, youth and the mediterranean diet in Spain. Development of kidmed, mediterranean diet quality index in children and adolescents. Public Health Nutrition 2004; 7: 931-935.
27. Martínez Mi, Hernández MD, Ojeda M, Mena R, Alegre A, Alfonso JL. Desarrollo de un programa de educación nutricional y valoración del cambio de hábitos alimentarios saludables en una población de estudiantes de enseñanza secundaria obligatoria. Nutr Hosp 2009; 24: 504-510.

28. KIm S, HaInes PS, Siega-Riz AM, Popkin BM. The Diet Quality Index-International (DQI-I) provides an effective tool for cross-national comparison of diet quality as illustrated by China and the United States. J Nutr 2003; 133: 3476-3484.

29. Tur JA, Romaguera D, Pons A. The diet quality index-international (DQI-I): is it a useful tool to evaluate the quality of the mediterranean diet? Br J Nutr 2005; 93: 369-376.

30. González CA, Argilaga S, Agudo A, Amiano P, Barricarte A, BeguiristáIn JM et al. Diferencias sociodemográficas en la adhesión al patrón de dieta mediterránea en poblaciones de España. Gac Sanit 2002; 16: 214-221.

31. Tur JA, Romaguera D, Pons A. Adherence to the mediterranean dietary pattern among the population of the balearic islands. $\mathrm{Br} \mathrm{J}$ Nutr 2004; 92: 341-346.

32. Serra-Majem Li, Ribas L, García A, Pérez-Rodrigo C, ARANCETA J. Nutrient adequacy and mediterranean diet in spanish school children and adolescents. Eur J Clin Nutr 2003; 57 (Suppl 1): S35-S39.

33. López J, Gómez P, Castro P, Marín C, Paz E, Bravo $\mathrm{MD}$ et al. La dieta mediterránea mejora la resistencia a la oxidación de las lipoproteínas de baja densidad (LDL). Med Clin (Barc) 2000; 115: 361-365.

34. Guerra A, Feldl F, Koletzko B. Fatty acid composition of plasma lipids in healthy Portuguese children: is the mediterranean diet disappearing? Ann Nutr Metab 2001; 45: 78-81.

35. Schmidhuber J. The european diet - evolution, evaluation and impacts of the CAP. Organización de las Naciones Unidas para la Agricultura y la Alimentación (FAO) 2008.

36. Nicklas TA, Reger C, Myers, O`Neil C. Breakfast consumption with and without vitamin-mineral supplement use favourably impacts daily nutrient intake of ninth-grade students. J Adolesc Health 2000; 27: 314-321.

37. DurÁ T. El desayuno de los alumnos de educación secundaria obligatoria (ESO). Nutr Hosp 2002; 17: 189-196. 